

\title{
Mode group specific amplification length in an asymmetric LPG assisted few-mode EDFA
}

Vipul Rastogi, Ankita Gaur, Pierre Aschieri, Bernard Dussardier

\section{To cite this version:}

Vipul Rastogi, Ankita Gaur, Pierre Aschieri, Bernard Dussardier. Mode group specific amplification length in an asymmetric LPG assisted few-mode EDFA. Optics Communications, 2017, 382, pp.13 17. 10.1016/j.optcom.2016.07.060 . hal-01390880

\section{HAL Id: hal-01390880 https://hal.science/hal-01390880}

Submitted on 7 Nov 2016

HAL is a multi-disciplinary open access archive for the deposit and dissemination of scientific research documents, whether they are published or not. The documents may come from teaching and research institutions in France or abroad, or from public or private research centers.
L'archive ouverte pluridisciplinaire HAL, est destinée au dépôt et à la diffusion de documents scientifiques de niveau recherche, publiés ou non, émanant des établissements d'enseignement et de recherche français ou étrangers, des laboratoires publics ou privés. 


\title{
Mode group specific amplification length in an
} asymmetric LPG assisted few-mode EDFA

\author{
${ }^{1}$ Vipul Rastogi, ${ }^{1 *}$ Ankita Gaur, ${ }^{2}$ Pierre Aschieri and ${ }^{2}$ Bernard Dussardier \\ ${ }^{I}$ Department of Physics, Indian Institute of Technology Roorkee, Roorkee 247667, INDIA \\ ${ }^{2}$ Université Nice Sophia Antipolis, CNRS, LPMC, UMR 7336, 06108 Nice CEDEX 2, France
}

*Corresponding author: ankitagaur.phy@gmail.com

This article presents a scheme for few-mode EDFA, which allows to choose independent amplification lengths for different mode groups. The EDF is a dual concentric core fiber, where the central core is connected to the line FMF and the ring core is doped with erbium to provide amplification. The modes of FMF are launched into the central core of the EDF, are converted into ring modes using LPG for amplification and then converted back into central core modes using another LPG. The distance between the LPGs determines the amplification length. The amplification length, can thus, be chosen for a given mode group. We demonstrate the working of this concept by choosing $\mathrm{LP}_{11}$ and $\mathrm{LP}_{21}$ mode groups of the FMF and show that a suitable choice of amplification lengths for the two mode groups can tailor the differential modal gain (DMG) to any desired value. We demonstrate achieving zero DMG among all the mode of $\mathrm{LP}_{11}$ and $\mathrm{LP}_{21}$ mode groups using this concept while having gain in excess of $20 \mathrm{~dB}$. The study should be useful for optical fiber communication system employing space-division multiplexing (SDM).

Key words: Long Period Grating, Erbium-doped fiber amplifier, Few mode fiber, Dual-core fiber, Space Division Multiplexing. 


\section{Introduction}

Presently, the high capacity telecommunication systems rely on components and devices based on single mode optical fibers. Thanks to succeeding technological breakthroughs, the trend in terms of capacity growth has been almost ten times every four years [1]. However, projections indicate that the large difference in growth rates between the fiber capacity and the traffic demand is expected to soon create a shortage.

In this context, the Space Division Multiplexing (SDM) based on multi core fibers (MCF) or few mode fibers (FMF) [2] is an attractive concept that needs a total revision of the paradigm of optical links, including all components and digital signal processing. It is out of the scope of this paper to compare the respective advantages of FMF- and MCF-based systems. Both technologies shall eventually contribute to the increasing of the global data rate. They can also be integrated in hybrid systems using few-mode core fiber [3] or a combination of FMF and MCF [4].

A key component of optical communication system is EDFA [5]. So, it is necessary to design few-mode erbium doped fiber amplifier (FMEDFA) [6-10] for FMF-SDM system [1112]. The principal requirements are that the system provides equal power and SNR among all mode groups during propagation and at the receiver, and that crosstalk is minimized in-line and at repeaters. This imposes that the differential modal gain (DMG) be carefully controlled to compensate for the differential modal loss along the passive FMF span between repeaters. Several FMEDFA have been reported to enable FMF-based SDM communication systems $[13,14]$. A few-mode amplifier compatible with 6-mode SDM communication has been studied experimentally [15]. A step-index fiber with alternate two erbium-doped regions (one annular, one central) [16] has been studied to minimize DMG of four mode-groups (LP ${ }_{01}$, $\mathrm{LP}_{02}, \mathrm{LP}_{11}$, and $\left.\mathrm{LP}_{21}\right)$. However, once the fibre is manufactured, the control of DMG is only achievable through very precise tuning of the pumping scheme [17]. An improvement in 
DMG of two mode groups has been experimentally demonstrated using ring-core EDFA [18]. A six-spatial-mode EDFA employing a mode-selective photonic lantern has been studied experimentally with gain $\sim 16 \mathrm{~dB}$ and $\mathrm{DMG}<1 \mathrm{~dB}$ at $1550 \mathrm{~nm}$ [19]. A cladding pumped EDFA supporting four and six-mode-groups has been studied to minimize the DMG using Genetic Algorithm [20]. A cladding-pumped ten-mode fiber amplifier with 20-dB gain and 2dB DMG has been reported [21].

Alternatively, in order to avoid crosstalk between signal modes at the amplification stage, it is interesting to selectively amplify each mode group along a "virtual" amplifier that is optimised for this group. An interesting way is to design an EDF having specific amplifying zones, into which one selectively amplifies each mode group for a determined propagation length, i.e. a determined gain. We believe that it is achievable by implementing already available technologies. We numerically demonstrate the concept of an original FMEDFA. For the sake of clarity, we deliberately choose a simple situation : We propose an EDF scheme that equally amplifies 8 modes (including two polarizations and two orientations) of two mode-groups $\mathrm{LP}_{11}$ and $\mathrm{LP}_{21}$ input from an $\mathrm{FMF}$, with more than $20 \mathrm{~dB}$ of gain each. It may be noted that we have not chosen $\mathrm{LP}_{0 \mathrm{~m}}$ mode groups: although these mode groups could be implemented in our scheme in principle, they are less interesting for SDM due to their 2-fold degeneracy as compared to 4-fold degeneracy of other mode groups. The proposed EDF configuration employs a coupled concentric dual-core structure. The central core (core-1) is designed so that the input signal modes from the FMF line fiber are efficiently coupled into the EDF. Only the outer ring core (core-2) of the EDF is doped with erbium. The $\mathrm{LP}_{11}$ and $\mathrm{LP}_{21}$ mode groups of the FMF are injected into the corresponding core1 modes of the EDF. They are then converted into a set of mode groups in core- 2 using paired asymmetric long period gratings (ALPGs) that are separated by an optimized distance along the EDF. The asymmetric structure of the pairs of ALPGs allows for the conversion of 
any mode symmetry to any other, for example from $\mathrm{LP}_{11}$ to $\mathrm{LP}_{31}$ [22]. The core-2 mode groups are amplified along a specific distance and then converted back into core-1 modes. The core- 2 modes selected for amplification have nearly equal overlaps with the pump mode thus resulting in small DMG. Since the proposed configuration allows the flexibility of having a specific amplification length for each mode group, zero-DMG (or any other DMGvalue) can be achieved by choosing optimized amplification lengths. As a proof of concept we present here an optimum combination of EDF lengths for various mode groups to obtain net zero DMG for all the polarizations and orientations in the $\mathrm{LP}_{11}$ and $\mathrm{LP}_{21}$ mode groups. We study the performance of the FMEDFA using two different pumping configurations of the erbium-doped ring core (core-2): all pump power injected into one ring mode, or equally injected into all the pump modes of the EDF. We show that this does not affect the DMG after APLG inter-distance optimization. However the second case is less efficient as only that fraction of pump power which is confined in core- 2 is used up for amplification with the proposed configuration. We numerically demonstrate more than $20 \mathrm{~dB}$ gain at $1530 \mathrm{~nm}$ wavelength with zero DMG. Other values of gain and DMG are achievable by design. Although the present work is limited to only two mode groups and a single wavelength channel, it may be extended to several mode groups and many wavelength channels using suitably designed ALPGs.

\section{Fiber Design and Working Principle}

The proposed FMEDFA is composed of line FMF, an original few mode EDF, and paired ALPGs. Fig1(a) shows the schematic line FMF, consisting of a step index core (radius a) and index difference $\Delta n_{1}$. The proposed dual concentric core EDF structure is schematically shown in Fig. 1(c). It consists of a central core $(r<a)$ with index difference $\Delta n_{1}$ and a ring core $(\mathrm{b}<r<c)$ with relative index difference $\Delta n_{2}$ with respect to pure silica cladding. The ring core is doped with $\mathrm{Er}^{3+}$ ions (blue-shaded region) and works as the 
amplifying core. For the sake of clarity, LP mode-groups are often referred to as LP modes. Figs. 1 (b) and (d) show the mode intensity profiles of FMF and FMEDF respectively. The value of various fiber parameters used are $\Delta n_{1}=0.018, \Delta n_{2}=0.02, a=4.5 \mu \mathrm{m}, b=6 \mu \mathrm{m}$ and $c=9 \mu \mathrm{m}$ at $1530-\mathrm{nm}$ wavelength. These parameters are chosen so that in the wavelength range of interest there is no resonance between the central core and the ring core for the desired sets of modes. There is sufficient mode spacing between the effective indices ( $>$ $5 \times 10^{-4}$ ) of the modes to avoid mode coupling due to micro-bending [23]. The schematic of the proposed amplifier is shown in Fig.1(e). Among the mode shown in Fig.1(e), LP 12,EDF $_{\text {and }}$ $\mathrm{LP}_{22 \text {,EDF }}$ can be identified as core mode as their energy is mostly confined in the central core, whereas $\mathrm{LP}_{21, \mathrm{EDF}}$ and $\mathrm{LP}_{31, \mathrm{EDF}}$ have their energy mostly confined in the ring and can be identified as ring modes. We can also see that the $\mathrm{LP}_{12, \mathrm{EDF}}$ and $\mathrm{LP}_{22, \mathrm{EDF}}$ mode intensity profiles resemble and would have good overlap with those of the $\mathrm{LP}_{11, \mathrm{FMF}}$ and $\mathrm{LP}_{21, \mathrm{FMF}}$ line modes, respectively. The $\mathrm{LP}_{11, \mathrm{FMF}}$ and $\mathrm{LP}_{21, \mathrm{FMF}}$ mode of the FMF excite the $\mathrm{LP}_{12, \mathrm{EDF}}$ and $\mathrm{LP}_{22, \mathrm{EDF}}$ modes of EDF. The fractional power coupled from $\mathrm{LP}_{11, \mathrm{FMF}}$ to the modes other than $\mathrm{LP}_{12, \mathrm{EDF}}$ remains less than 0.1 and from $\mathrm{LP}_{21, \mathrm{FMF}}$ to the modes other than $\mathrm{LP}_{22, \mathrm{EDF}}$ remains less than 0.04. We use ALPGs to couple light from the $\mathrm{LP}_{12, \mathrm{EDF}}$ and $\mathrm{LP}_{22, \mathrm{EDF}}$ core modes to the $\mathrm{LP}_{21, \mathrm{EDF}}$ and $\mathrm{LP}_{31, \mathrm{EDF}}$ ring modes for amplification, respectively. After amplification, these ring modes couple back into core modes via the ALPGs. We choose the $\mathrm{LP}_{02, \mathrm{EDF}, \mathrm{p}}$ pump mode of EDF for amplification, which can be excited by standard techniques using phase mask or spatial light modulator. The $\mathrm{LP}_{02, \mathrm{EDF}, \mathrm{p}}-\mathrm{LP}_{21, \mathrm{EDF}}$ mode overlap and $\mathrm{LP}_{02, \mathrm{EDF}, \mathrm{p}}-\mathrm{LP}_{31, \mathrm{EDF}}$ mode overlaps are $5.24 \times 10^{9}$ and $5.34 \times 10^{9}$, respectively. Nearly equal overlaps with signal mode groups help in achieving small DMG. The length of the amplifier for a specific mode group is decided by the distance between the in-coupling and out-coupling paired ALPGs and therefore the two mode groups can have independent amplifier lengths. A suitable choice of 
amplifier lengths for the two mode groups is used to achieve zero DMG, or any desired DMG value.

\section{Mode Conversion using ALPG}

A set of two identical ALPGs namely LPG-1 and LPG-2 (Fig.1(e)) couple light from $\mathrm{LP}_{12, \mathrm{EDF}}$ to $\mathrm{LP}_{21, \mathrm{EDF}}$ and back, respectively. Another set LPG-3 and LPG-4 couple light between $\mathrm{LP}_{22, \mathrm{EDF}}$ and $\mathrm{LP}_{31, \mathrm{EDF}}$ modes. The index modulation in the ALPGs is defined as

$$
\Delta n^{2}(r, \phi, z)=\left\{\begin{array}{ll}
\Delta n_{0}^{2} \sin (K z) & \text { for } 0<\phi<\pi, \forall r \\
0 & \text { elsewhere }
\end{array}\right\}
$$

where $\Delta n_{0}^{2}$ is the amplitude of modulation in $n^{2}(r, \phi, z)$ and $K=2 \pi / \Lambda, \Lambda$ being the grating period, $r$ the radius and $\phi$ the azimuthal angle. In our calculations we have set the amplitude of refractive index modulation at $\Delta n_{0}=3 \times 10^{-4}$, which can be achieved by $\mathrm{CO}_{2}$ laser writing technology for coupling between circularly asymmetric modes of the fiber [22].

As a result, the longitudinal variation of the amplitude in the $m^{\text {th }}$ mode of the fiber can be written as:

$$
\frac{d A_{m}}{d z}=-i \sum_{n=1}^{N} C_{m n} A_{n}(z) \exp \left(i \Delta \beta_{m n} z\right) \sin (K z)
$$

where $N$ is the total number of modes supported by the fiber. $\Delta \beta_{\mathrm{mn}}$ is the phase mismatch between the $m^{\text {th }}$ and $n^{\text {th }}$ modes of the fiber and is defined as $\Delta \beta_{\mathrm{mn}}=2 \pi\left(n_{\text {eff,m }}-n_{\text {eff, },}\right) / \lambda_{0}$, where $\lambda_{0}$ is the free space wavelength, and $n_{\text {eff,i }}(\mathrm{i}=\mathrm{m}, \mathrm{n})$ are the effective indices of the $i^{\text {th }}$ mode. The effective indices of the modes and the corresponding modal field profiles $\psi_{\mathrm{i}}(r, \phi)$ of the fiber have been evaluated by using the transfer matrix method [24]. $C_{m n}$ are the coupling coefficients between the $m^{\text {th }}$ and $n^{\text {th }}$ modes of the fiber and are given by the following expression: 


$$
C_{m n}=\frac{k_{0}}{2 n_{e f f}^{m}} \frac{\int_{0}^{2 \pi} \int_{0}^{\infty} \psi_{m}^{*} \Delta n^{2}(r, \phi) \psi_{n} r d r d \phi}{\int_{0}^{2 \pi} \int_{0}^{\infty} \psi_{m}^{*} \psi_{m} r d r d \phi}
$$

The system of $N$ coupled equations represented by Eq. (2) has been solved by using the Runge-Kutta method, and the power of each mode as a function of propagation distance has been evaluated. Even $(\cos l \phi)$ and odd $(\sin l \phi)$ core modes are coupled to their respective orientations of ring modes through the ALPGs. In general the coupling coefficients in both cases are not the same and one has to adjust the grating length in order to achieve the same coupling for even and odd core modes. For coupling from $\mathrm{LP}_{12, \mathrm{EDF}}$ to $\mathrm{LP}_{21, \mathrm{EDF}}$ mode via LPG1, the grating period is $\Lambda_{1}=479 \mu \mathrm{m}$ and the coupling length is $L_{\mathrm{cl}}=4.3 \mathrm{~cm}$. LP 22 ,EDF to $\mathrm{LP}_{31, \mathrm{EDF}}$ mode conversion through LPG-3 requires a grating period $\Lambda_{2}=198 \mu \mathrm{m}$, and the grating length $L_{\mathrm{c} 2}=9.6 \mathrm{~cm}$. We have also verified that the coupling of power due to LPGs among any other set of well guided modes in the EDF remains less than $-20 \mathrm{~dB}$. It means there is no undesired mode coupling due to LPG.

\section{Gain Modeling}

The two mode groups of the FMF, $\mathrm{LP}_{11, \mathrm{FMF}}$ and $\mathrm{LP}_{21, \mathrm{FMF}}$ with even and odd orientations and two polarizations each, form 8 SDM channels. The input signal power in each orientation and polarization of signal mode groups has been chosen as $30 \mu \mathrm{W}$. These mode groups of the FMF excite the $\mathrm{LP}_{12, \mathrm{EDF}}$ and $\mathrm{LP}_{22, \mathrm{EDF}}$ mode groups of the EDF. The mode mismatch loss between $\mathrm{LP}_{11, \mathrm{FMF}}$ and $\mathrm{LP}_{12, \mathrm{EDF}}$ is $1.02 \mathrm{~dB}$ and that between $\mathrm{LP}_{21, \mathrm{FMF}}$ and $\mathrm{LP}_{22, \mathrm{EDF}}$ is $1.43 \mathrm{~dB}$. The mode conversion loss due to ALPG from $\mathrm{LP}_{12, \mathrm{EDF}}$ to $\mathrm{LP}_{21, \mathrm{EDF}}$ is 1.25 $\mathrm{dB}$ and that for conversion from $\mathrm{LP}_{22, \mathrm{EDF}}$ to $\mathrm{LP}_{31, \mathrm{EDF}}$ is $0.65 \mathrm{~dB}$. The powers of $\mathrm{LP}_{21, \mathrm{EDF}}$ and $\mathrm{LP}_{31, \mathrm{EDF}}$ mode groups of the EDF are mostly confined in the ring, erbium-doped core. For calculating the gain we have slightly modified the model described by $\mathrm{N}$. Bai et al. by 
incorporating backward ASE. For details of the model, readers are referred to Ref. [17]. For efficient pump absorption we choose the $\mathrm{LP}_{02, \mathrm{EDF}, \mathrm{p}}$ mode at $980 \mathrm{~nm}$ wavelength.

The variation of gain and DMG of the FMF mode groups including all losses due to mode coupling and mode conversion versus amplifier length (assuming equally spaced paired ALPGs for both mode groups) is shown in Figs. 2(a) and 2(b), respectively, for $150 \mathrm{~mW}$ pump power. We can see that both mode groups have more than $20 \mathrm{~dB}$ of gain for a spacing between paired-ALPG longer than $17.8 \mathrm{~m}$. Also in the case of an equal distance between paired ALPGS (LPG-1/LPG2 and LPG-3/LPG-4), the DMG is less than $0.69 \mathrm{~dB}$. Also there is no DMG between the two orientations within a same mode group because the overlaps of the even and the odd orientations of signal modes with the pump mode are equal.

In Fig. 2(a), we see that a choice of the same amplifier length of $17.8 \mathrm{~m}$ for both mode groups results in approximately $20 \mathrm{~dB}$ of gain, with $0.69 \mathrm{~dB}$ DMG. This residual DMG can be made equal to zero by choosing optimum amplifier lengths $L_{1}$ and $L_{2}$ for the $\mathrm{LP}_{11 \text {,FMF }}$ and $\mathrm{LP}_{21 \text {,FMF }}$ mode groups, respectively. A choice of $L_{1}=17.8 \mathrm{~m}$ and $L_{2}=16.6 \mathrm{~m}$ leads to zeroDMG at $150 \mathrm{~mW}$ pump power. For this combination of $L_{1}$ and $L_{2}$ and if the pump power is increased, then a little amount of DMG appears, as shown in Fig. 3(a). It is therefore required to have different combination of $L_{1}$ and $L_{2}$ to have zero-DMG at different pump powers. For practical purposes and to minimize the complexity it is better to have as small length difference $\Delta L$ as possible between $L_{1}$ and $L_{2}$. In order to have an estimate of $\Delta L$, we have plotted the variation of $\Delta L$ with pump power corresponding to $20 \mathrm{~dB}$ of gain in Fig. 3(b). We see that for $20 \mathrm{~dB}$ gain, zero DMG can be achieved by either having low pump power and large $\Delta L$; or by having high pump power and small $\Delta L$. At $330 \mathrm{~mW}$ of pump power, $\Delta L$ is as small as $0.54 \mathrm{~m}$.

We next studied the effect of equally exciting all the supported pump mode of the EDF. The fiber supports 23 mode groups at pump wavelength. A total of $168 \mathrm{~mW}$ power was 
evenly injected in these modes altogether. The variation of gain and DMG (assuming equally spaced ALPGs pairs) is shown in Fig.4. We can see more than $20 \mathrm{~dB}$ of gain for two mode groups for fiber length longer than $20.7 \mathrm{~m}$ and DMG below $0.71 \mathrm{~dB}$. The optimization of the DMG is accessible by optimization of the amplifiers lengths $L_{1}$ and $L_{2}$. Although this pumping configuration necessitates a longer EDF length, this shows that the selective excitation of $\mathrm{LP}_{02, \mathrm{EDF}, \mathrm{p}}$ pump mode is not critical. As long as the pump power in the ring is uniform, the DMG would be small. However, the utilization of the pump power would not be efficient as the power in the central core would not contribute to amplification.

In the simulations, we have not considered amplification of $\mathrm{LP}_{01}$ mode group as it is least interesting for SDM due to its two-fold degeneracy. However, if required it can also be amplified by coupling it to a suitable ring mode and DMG can be controlled by using another set of gratings. To consider $\mathrm{LP}_{01}$ mode group it has to be converted into the $\mathrm{LP}_{02, \mathrm{EDF}}$ mode using symmetric LPG of period $610 \mu \mathrm{m}$. The DMG obtained while considering the $\mathrm{LP}_{01}$, $\mathrm{LP}_{11}$ and $\mathrm{LP}_{21}$ mode groups is $\sim 1 \mathrm{~dB}$.

Although the design proposed in the present paper has been demonstrated only at one wavelength, it can be extended to a wavelength division multiplexed (WDM) mode of operation in the entire C-band. For this, one would have to slightly chirp the paired ALPGs. We have calculated that the variation required in grating period along the grating length for $\mathrm{LP}_{12, \mathrm{EDF}} \leftrightarrow \mathrm{LP}_{21, \mathrm{EDF}}$ mode conversion is $2.5 \%$ and that for $\mathrm{LP}_{22, \mathrm{EDF}} \leftrightarrow \mathrm{LP}_{31, \mathrm{EDF}}$ mode conversion is $0.5 \%$ in the entire C-band. It is therefore possible to extend the design to WDM systems by using slightly chirped or concatenated gratings and adequate gain flattening filters in the wavelength domain. 


\section{Conclusion}

We have proposed a novel scheme for amplification of $\mathrm{LP}_{11}$ and $\mathrm{LP}_{21}$ mode groups of a FMF for applications in SDM optical communication system. Our numerically simulated results have shown that an ALPG-assisted dual-concentric core EDF can help in obtaining potentially zero DMG, or any desired value of DMG, thanks to the independent choice of amplifier lengths of the signal mode groups. This original concept can in principle be scaled to more mode groups, and further designed to operate in WDM.

\section{References}

1. D. J. Richardson, J. M. Fini, and L. E. Nelson, Space-division multiplexing in optical fibers, Nat. Photonics 7(2013) 354-362.

2. R. J. Essiambre, R. Ryf, N. K. Fontaine, and S. Randel, Breakthroughs in Photonics 2012: Space-Division Multiplexing in Multimode and Multicore Fiber for HighCapacity Optical Communication, IEEE Photonics Journal 5(2013) 0701307.

3. D. Qian, E. Ip, M.-F. Huang, M.-J. Li, A.Dogariu, S. Zhang, Y. Shao, Y.-K. Huang, Y. Zhang, X. Cheng, Y. Tian, P. Nan Ji, A. Collier, Y. Geng, J. Liñares, C. Montero, V. Moreno, X. Prieto, and T. Wang, 1.05Pb/s Transmission with 109b/s/Hz Spectral Efficiency using Hybrid Single- and Few-Mode Cores, Proceeding of Frontiers In Optics, Rochester, New York, United States (2012) Paper FW6C.3.

4. N. K. Fontaine, T. Haramaty, R. Ryf, H. Chen, L. Miron, L. Pascar, M. Blau, B. Frenkel, L. Wang, Y. Messaddeq, S. LaRochelle, R. J. Essiambre, Y. Jung, Q. Kang, J. K. Sahu, S. U. Alam, D. J. Richardson and D. M. Marom, Heterogeneous SpaceDivision Multiplexing and Joint Wavelength Switching Demonstration, Proceeding of Optical Fiber Communication Conference, Postdeadline Paper, Los Angeles, California, United States (2015) Paper TH5C.5. 
5. C. R. Giles, and E. Desurvire, Modeling Erbium-Doped Fiber Amplifiers, J. Lightwave Technol. 9(1991) 271-283.

6. E. Ip, Gain Equalization for few-Mode Fiber Amplifiers Beyond Two Propagating Mode Groups, IEEE Photon. Technol. Lett. 24(2012) 1933-1936.

7. N. Bai, E. Ip, Y. K. Huang, E. Mateo, F. Yaman, M. J. Li, S. Bickham, S. Ten, J. Liñares, C. Montero, V. Moreno, X. Prieto, V. Tse, K. M. Chung, A. P. T. Lau, H. Y. Tam, C. Lu, Y. Luo, G. D. Peng, G. Li, T. Wang, Mode-division multiplexed transmission with inline few-mode fiber amplifier, Opt. Express 20(2012) 26682680.

8. G. Le Cocq, Y. Quiquempois, A. Le Rouge, G. Bouwmans, H. El Hamzaoui, K. Delplace, M. Bouazaoui, and L. Bigot, Few mode $\mathrm{Er}^{3+}$-doped fiber with microstructured core for mode division multiplexing in the C-band, Opt. Express 21(2013) 31646-31659.

9. D. Askarov, and J. M. Kahn, Design of Transmission Fibers and Doped Fiber Amplifiers for Mode-Division Multiplexing, IEEE Photon. Technol. Lett. 24 (2012) 1945-1948.

10. C. Jin, Y. Messaddeq, and S. LaRochelle, Tailored modal gain in a multi-mode erbium-doped fiber amplifier based on engineered ring doping profile, Proceeding of Photonics North, Ottawa, Canada (2013) 89150A1-89150A12.

11. V. A. J. M. Sleiffer, Y. Jung, V. Veljanovski, R. G. H. van Uden, M. Kuschnerov, Q. Kang, L. Grüner-Nielsen, Y. Sun, D. J. Richardson, S. Alam, F. Poletti, J. K. Sahu, A. Dhar, H. Chen, B. Inan, A. M. J. Koonen, B. Corbett, R. Winfield, A. D. Ellis, and H. de Waardt, $73.7 \mathrm{~Tb} / \mathrm{s}(96 \times 3 \times 256-\mathrm{Gb} / \mathrm{s})$ mode-division-multiplexed DP16QAM transmission with inline MM-EDFA, Opt. Express, 20(2012) B428-B438. 
12. R. Ryf, S. Randel, A. H. Gnauck, C. Bolle, A. Sierra, S. Mumtaz, M. Esmaeelpour, E. C. Burrows, R. J. Essiambre, P. J. Winzer, D. W. Peckham, A. H. McCurdy, and R. Lingle, Mode-Division Multiplexing Over $96 \mathrm{~km}$ of Few-Mode Fiber Using Coherent $6 \times 6$ MIMO Processing, J. Light Technol. 30(2012) 521-531.

13. P. Genevaux, C. Simonneau, and G. Charlet, Challenges in the Design of Few Mode EDFAs, Proceeding of Optical Fiber Communication Conference, Anaheim, California, United States (2016) Paper Tu2I.2.

14. E. L. Lim, Y. Jung, Q. Kang, T. C. May-Smith, N.H.-L. Wong, R. Standish, F. Poletti, J.K. Sahu, S. Alam, and D.J. Richardson, First Demonstration of Cladding Pumped Few-moded EDFA for Mode Division Multiplexed Transmission, Proceeding of Optical Fiber Communication Conference, San Francisco, California, United States (2014), Paper M2J.2.

15. M. Salsi, Challenges of Few Mode Amplifiers, Proceeding of Optical Fiber Communication Conference, San Francisco, California, United States (2014) Paper Tu2D.2.

16. Q. Kang, E. Lim, Y. Jung, F. Poletti, Shaif-ul Alam and D. J. Richardson, Design of Four-Mode Erbium Doped Fiber Amplifier with Low Differential Modal Gain for Modal Division Multiplexed, Proceeding Of Optical Fiber Communication Conference/ National Fiber Otics Engineers Conference, Anaheim, California, United States (2013) Paper OTu3G.

17. N. Bai, E. Ip, T. Wang' and G. Li, Multimode fiber amplifier with tunable modal gain using a reconfigurable multimode pump, Opt. Express 19(2011) 1660116611. 
18. H. Ono, T. Hosokawa, K. Ichii, S. Matsuo, and M. Yamada, Improvement of differential modal gain in few-mode fibre amplifier by employing ring-core erbiumdoped fibre, Electron. Lett. 51(2015) 172-173.

19. G. Lopez-Galmiche, Z. S. Eznaveh, J. E. Antonio-Lopez, A. M. V. Benitez, J. R. Asomoza, J. J. S. Mondragon, C. Gonnet, P. Sillard, G. Li, A. Schülzgen, C. M. Okonkwo, and R. A. Correa, Few-mode erbium-doped fiber amplifier with photonic lantern for pump spatial mode control, Opt. Lett. 41(2016) 2588-2591.

20. Q. Kang, E-L Lim, F. Poletti Y. Jung, C. Baskiotis, S. Alam, and D. J. Richardson, Minimizing differential modal gain in cladding pumped EDFAs supporting four and six mode groups, Opt. Express 22 (2014) 21499-21507.

21. N. K. Fontaine, B. Huang, Z. Sanjabieznaveh, H. Chen, C. Jin, B. Ercan, A. Velázquez-Benetez, S. H. Chang, R. Ryf, A. Schülzgen, J. Carlos Alvarado, P. Sillard, C. Gonnet, E. Antonio-Lopez, and R. Amezcua Correa, Multi-mode Optical Fiber Amplifier supporting over 10 Spatial Modes, Proceeding of Optical Fiber Communication Conference, Postdeadline paper, Anaheim, California, United States (2016) Paper Th5A.4.

22. R. Slavik, Coupling to circularly asymmetric modes via long-period gratings made in standard straight fiber Opt. Comm. 275(2007) 354-362.

23. S. Ramachandran, J. W. Nicholson, S. Ghalmi, M. F. Yan, P. Wisk, E. Monberg, and F. V. Dimarcello, Light propagation with ultra large modal areas in optical fibers Opt. Lett. 31(2006) 1797-1799.

24. K. Morishita, Numerical Analysis of Pulse Broadening in Graded index Optical Fibers IEEE Transactions on Microwave Theory and Techniques 29(1981) $348-352$. 


\section{Figure Captions}

Fig 1. (a) Schematic of transverse cross section and refractive index profile of the line FMF, (b) Contour plot of all supported mode groups of line FMF at $1530 \mathrm{~nm}$ wavelength, (c) Schematic of transverse cross section and refractive index profile of the FMEDF ( blue-shaded portion: $\mathrm{Er}^{3+}$ doped ring core, grey -shaded portion: central core), (d) Contour plot of all supported mode groups of FMEDF at $1530 \mathrm{~nm}$ wavelength, and (e) Schematic of mode conversion and amplification of mode: details in the next.

Fig 2. (a) Variation of gains of $\mathrm{LP}_{11, \mathrm{FMF}}$ and $\mathrm{LP}_{21, \mathrm{FMF}}$ mode groups with the amplification length.

(b) Variation of DMG in case of equal amplification lengths corresponding to different mode groups. The pump power is $150 \mathrm{~mW}$ on $\mathrm{LP}_{02, \mathrm{EDF}, \mathrm{p}}$ mode.

Fig 3. (a) Variation of gain with pump power for $L_{1}=17.8 \mathrm{~m}\left(\mathrm{LP}_{11, \mathrm{FMF}}\right.$ mode group) and $L_{2}=16.6 \mathrm{~m}\left(\mathrm{LP}_{21, \mathrm{FMF}}\right.$ mode group). Gain and DMG are $20 \mathrm{~dB}$ and $0 \mathrm{~dB}$ at 150 $\mathrm{mW}$, respectively

(b) Variation of change in optimum lengths of EDF with pump power for equal gain $(20 \mathrm{~dB})$.

Fig 4. (a) Variation of gain of the $\mathrm{LP}_{11, \mathrm{FMF}}$ and $\mathrm{LP}_{21, \mathrm{FMF}}$ mode groups with the amplification length when all pump modes are equally excited

(b) Corresponding variation of DMG in case of equal amplification lengths corresponding to different mode groups. 

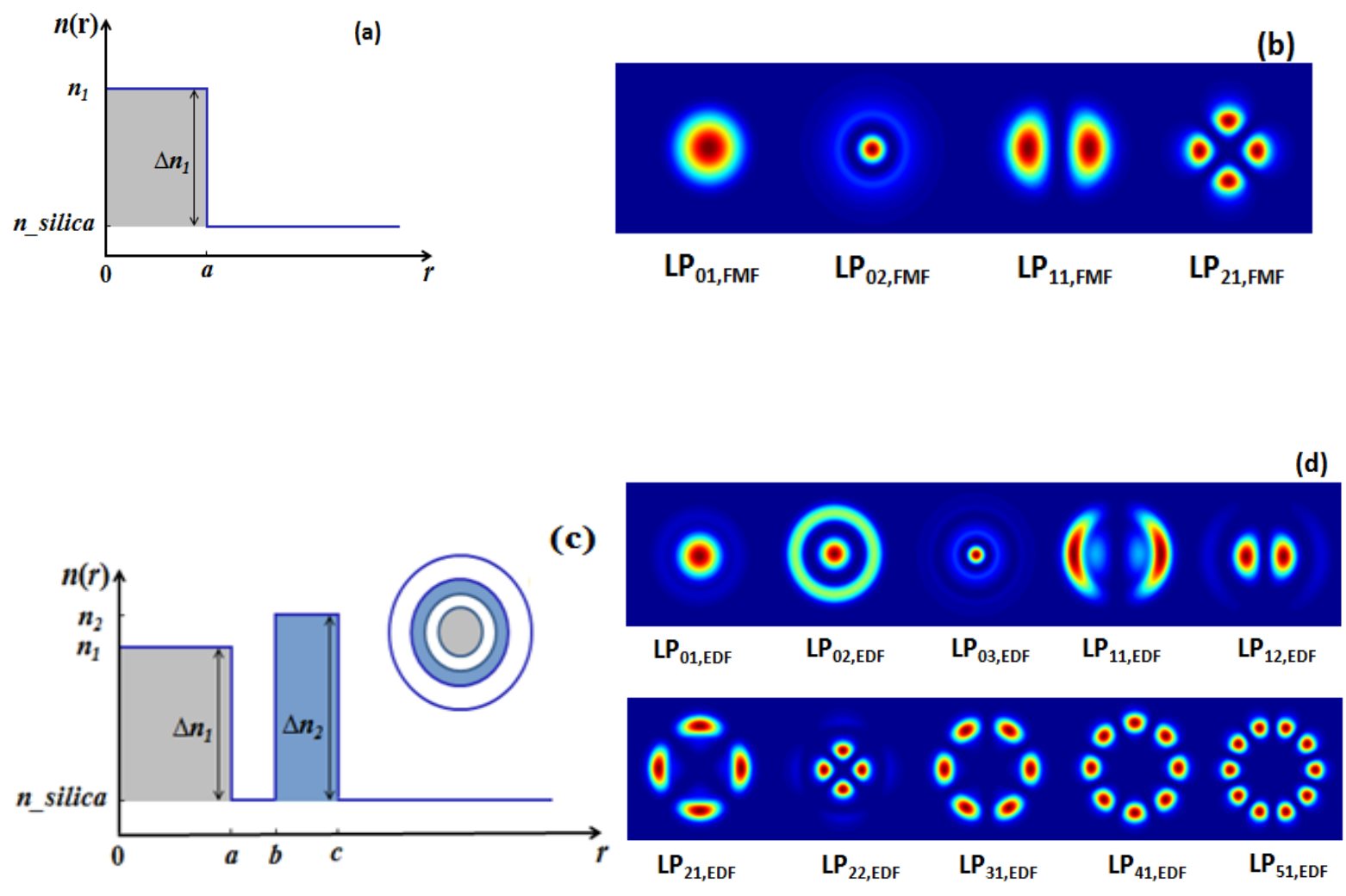

(c)

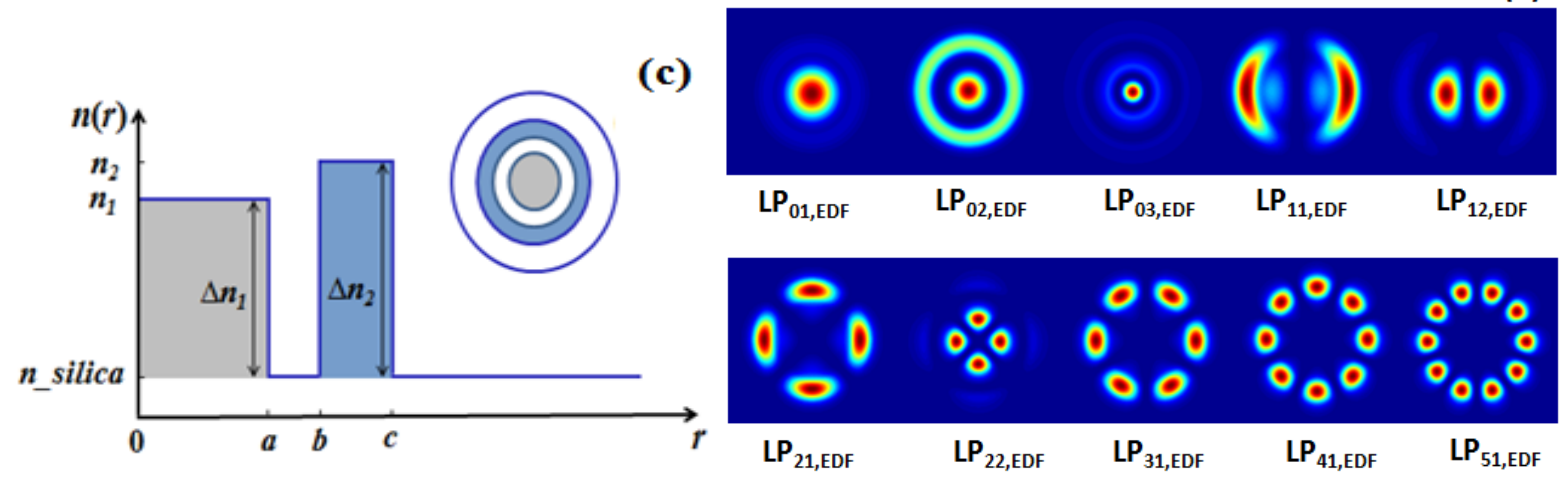

(d)

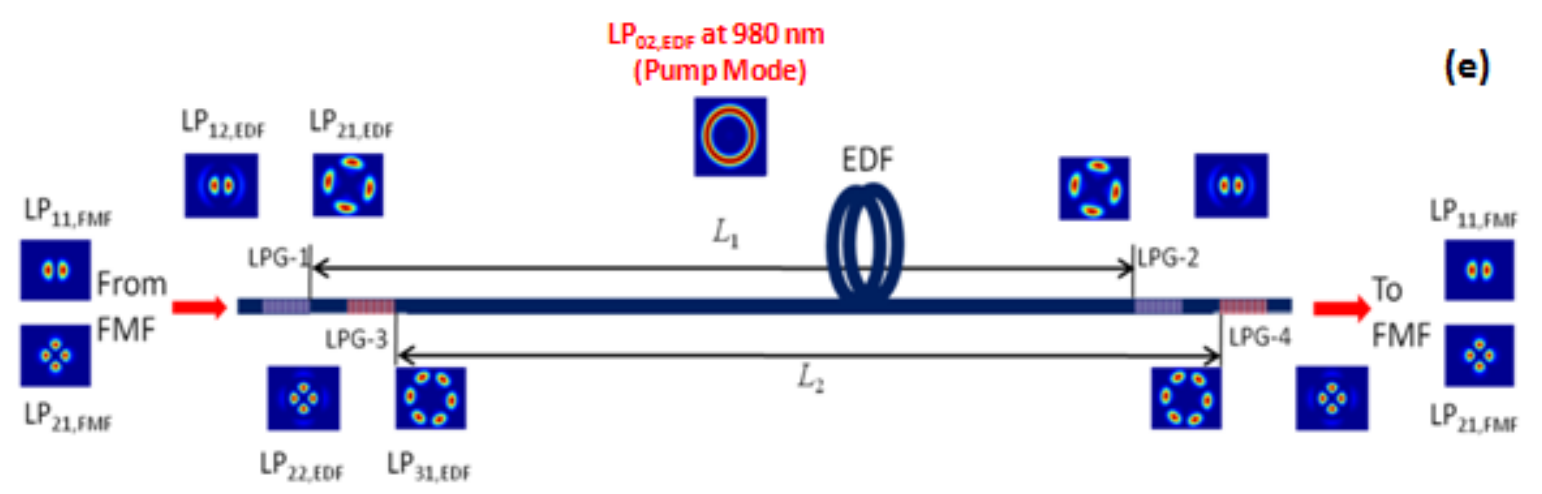

Fig.1 

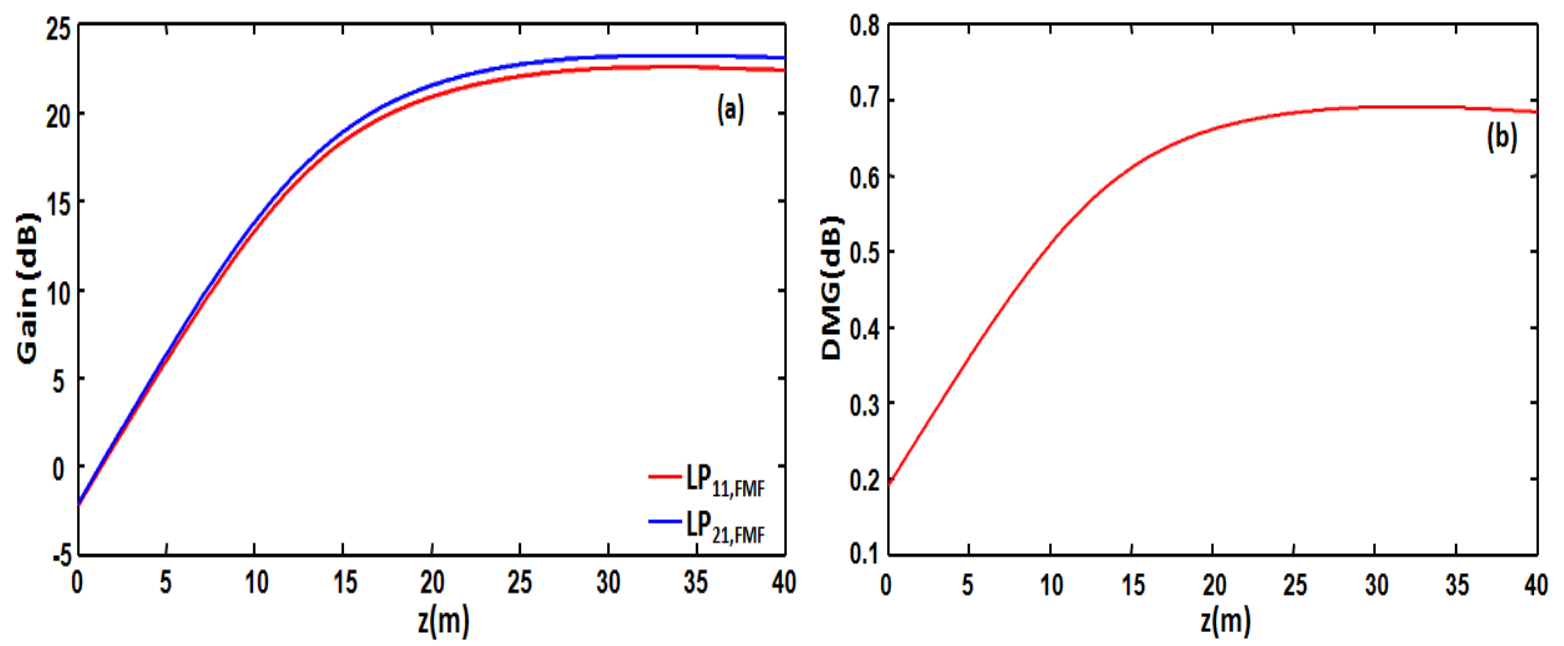

Fig.2
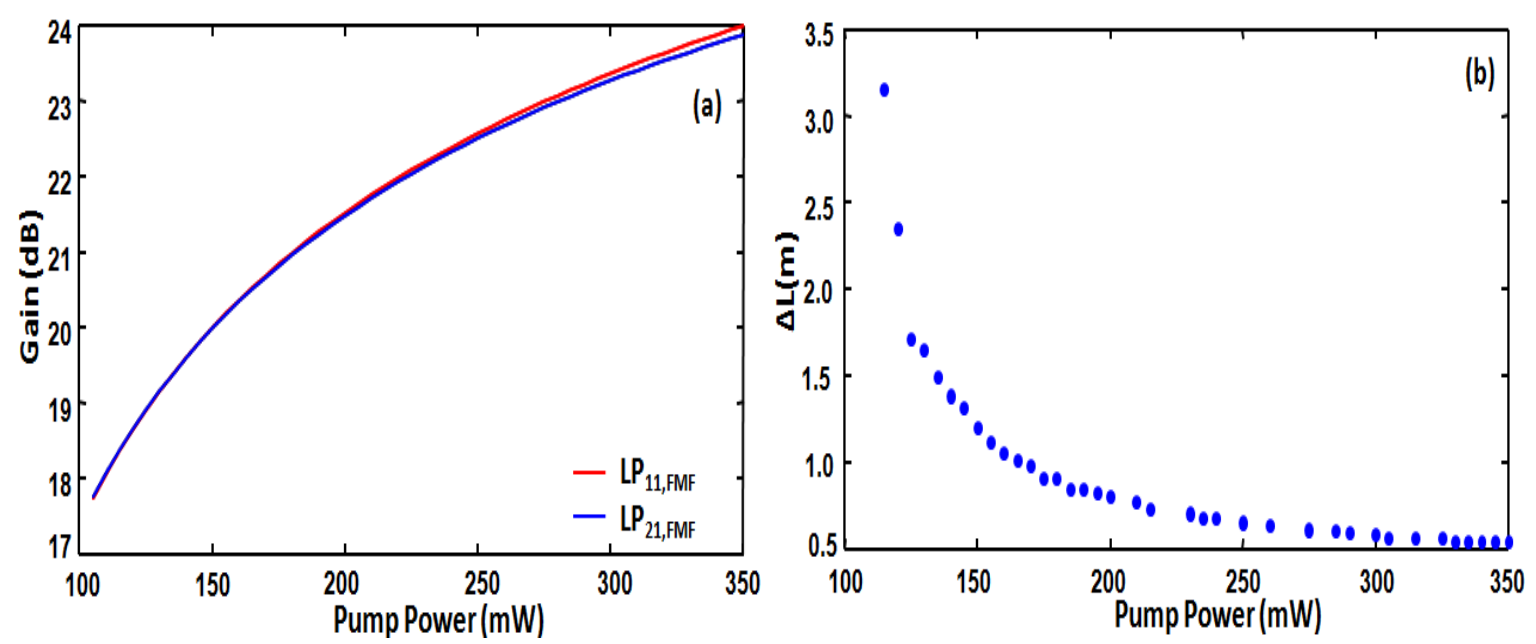

Fig.3
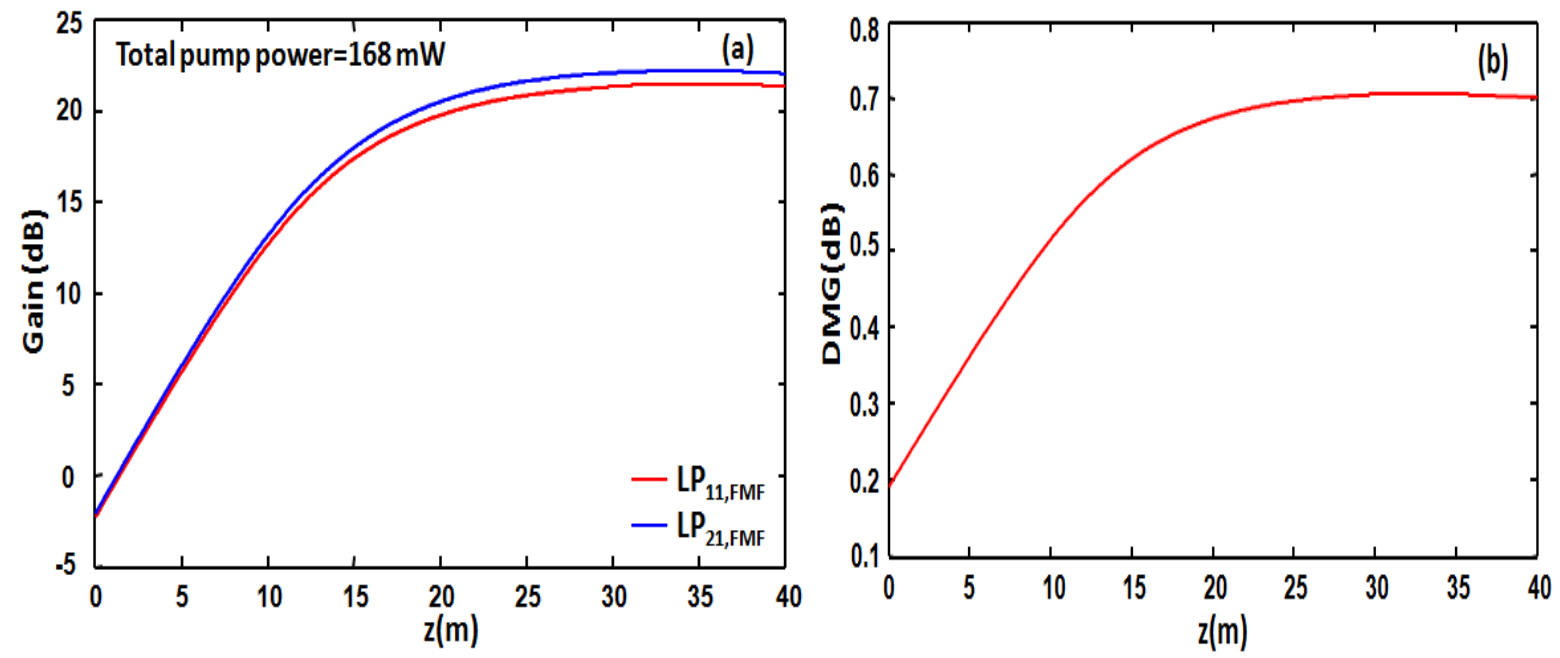

Fig.4 